\title{
Matching the termination of radiating non-uniform transmission-lines
}

\author{
R. Rambousky ${ }^{1}$, J. Nitsch ${ }^{2}$, and H. Garbe ${ }^{3}$ \\ ${ }^{1}$ Bundeswehr Research Institute for Protective Technologies and NBC Protection (WIS), Munster, Germany \\ ${ }^{2}$ Otto-von-Guericke University Magdeburg, Magdeburg, Germany \\ ${ }^{3}$ Leibniz University Hannover, Hannover, Germany \\ Correspondence to: R. Rambousky (ronald.rambousky@ieee.org)
}

\begin{abstract}
In this contribution a concept of matching the termination of radiating non-uniform transmission-lines is proposed. Using Transmission-Line Super Theory, position and frequency dependent line parameters can be obtained. Therefore, a characteristic impedance can be determined which is also position and frequency dependent. For a single wire transmission-line it could be shown that the maximum value of that characteristic impedance is an optimal termination in the sense of minimizing the variation of the current on the line. This indicates that matching is not a local effect at the position of the concentrated load but a cooperative process including the whole non-uniform transmission-line. In addition this choice of termination minimizes the variation of the radiated power over frequency.
\end{abstract}

\section{Introduction}

Usually transmission-lines (TL) are used in a matched state. A matched termination ensures that the reflection of forward traveling waves at the end of the line is minimized. This leads to a minimal variation of the current on the TL. In the classical transmission-line theory (TLT) matched termination is achieved by choosing the terminating impedance corresponding to the constant and real valued characteristic impedance of the classical TL. However, in many practical applications non-uniform wire structures are used. Instead of the mentioned classical TLT in such cases the exact theory in thin wire approximation, the so called TransmissionLine Super Theory (TLST), is intended to be used which includes particularly higher order modes and radiation effects. The transmission-line parameter matrix now is complex valued and a position and frequency dependent function. Therefore, it is a priori not clear which terminating impedance minimizes the reflections on the TL. This problem is analyzed using a simple but non trivial single wire non-uniform transmission-line. As an indicator of a good matching the current distribution along the wire at a fixed frequency is used. The effects to the frequency dependent variation of the current in the load and the variation of radiated power are also investigated.

\section{The investigated single wire non-uniform transmission-line}

For a single wire transmission-line the parameterization according to the arc length is a good choice. In the following work this paramter is named as $\zeta$. It is the so called natural parameter of the wire. When $L$ is the total arc length of the wire then $\zeta \in[0, L]$. Figure 1 shows the detailed geometry of the used non-uniform transmission-line. In reality this could be the principal structure (center wire) of a wire based open TEM-waveguide, such as a Nuclear Electromagnetic Pulse (NEMP) simulator. The horizontal line is part of the infinite PEC ground plane. The total arc length of the used TL is $1.609 \mathrm{~m}$. On the generator side there is a vertical riser of $10 \mathrm{~mm}$ length. The generator $(1 \mathrm{~V}, 50 \Omega)$ is placed between the beginning of the TL and the ground plane. The terminating impedance again is positioned between the end of the TL and the ground plane. Therefore, these terminating elements can actually be handled as boundary conditions when solving the TLST equation.

\section{Fundamentals of transmission-line super theory}

Transmission-line super theory (Haase and Nitsch, 2001; Haase et al., 2003; Haase, 2005; Nitsch et al., 2009; 




Fig. 1. Geometry and dimensioning of the used single wire nonuniform transmission-line

Nitsch and Tkachenko, 2010) is a full wave description of Maxwell's equations casted into the form of telegrapher's equations of classical TLT. Starting point is the parameterized representation of the TL in the thin-wire approximation (Tesche, 1972). The 3-dimensional curve $\boldsymbol{C}(\zeta)$ parameterized by its natural parameter, the arc length $\zeta$, describes the center line of the conductor. It has a circular cross section of the small radius $r_{0}$ and the total length $L$. Additionally the tangent vector $\boldsymbol{T}(\zeta)$ and the normal unit vector $\boldsymbol{N}^{u}(\zeta)$ are required for TLST. The TL is spanned over PEC ground. Therefore, the mirrored curve $\widetilde{\boldsymbol{C}}(\zeta)$ and the mirrored tangential vectors $\widetilde{\boldsymbol{T}}(\zeta)$ are needed to replace the PEC ground plane for further calculations.

The first TLST equation is derived from the continuity relation and is

$$
\frac{\partial}{\partial \zeta} i+j \omega q=0
$$

$q$ being the per-unit length charge and $i$ the current on the TL. The second TLST equation is derived from the Mixed Potential Integral Equation (MPIE) (Nitsch et al., 2009) and becomes

$$
\frac{\partial}{\partial \zeta} \int_{0}^{L} k_{c}\left(\zeta, \zeta^{\prime}\right) q\left(\zeta^{\prime}\right) d \zeta^{\prime}+j \omega \int_{0}^{L} k_{l}\left(\zeta, \zeta^{\prime}\right) i\left(\zeta^{\prime}\right) d \zeta^{\prime}=0 .
$$

Equation (2) describes the case where no distributed sources are present. The brief explanation of the TLST here is also restricted to a single wire TL for simplicity. The integral kernals $k_{c}$ and $k_{l}$ in the thin-wire approximation become

$$
\begin{aligned}
k_{c}\left(\zeta, \zeta^{\prime}, k\right) & =\frac{1}{\epsilon}\left[G\left(\zeta, \zeta^{\prime}, k\right)-\widetilde{G}\left(\zeta, \zeta^{\prime}, k\right)\right] \\
k_{l}\left(\zeta, \zeta^{\prime}, k\right) & =\mu\left[\boldsymbol{T}(\zeta) \cdot \boldsymbol{T}\left(\zeta^{\prime}\right) G\left(\zeta, \zeta^{\prime}, k\right)\right. \\
& \left.-\boldsymbol{T}(\zeta) \cdot \widetilde{\boldsymbol{T}}\left(\zeta^{\prime}\right) \widetilde{G}\left(\zeta, \zeta^{\prime}, k\right)\right] .
\end{aligned}
$$

$G\left(\zeta, \zeta^{\prime}, k\right)$ and $\widetilde{G}\left(\zeta, \zeta^{\prime}, k\right)$ are the Green's functions of the real and the mirrored wires in free space, respectively. To avoid a singularity in the Green's function for $\zeta=\zeta^{\prime}$ the observation point (described by the parameter $\zeta$ ) is shifted from the wire center in normal direction to the wire surface.

$G\left(\zeta, \zeta^{\prime}, k\right)=\frac{\exp \left(-j k\left|\boldsymbol{C}(\zeta)+\boldsymbol{N}^{u}(\zeta) r_{0}-\boldsymbol{C}\left(\zeta^{\prime}\right)\right|\right)}{4 \pi\left|\boldsymbol{C}(\zeta)+\boldsymbol{N}^{u}(\zeta) r_{0}-\boldsymbol{C}\left(\zeta^{\prime}\right)\right|}$
$\widetilde{G}\left(\zeta, \zeta^{\prime}, k\right)=\frac{\exp \left(-j k\left|\boldsymbol{C}(\zeta)+\boldsymbol{N}^{u}(\zeta) r_{0}-\widetilde{\boldsymbol{C}}\left(\zeta^{\prime}\right)\right|\right)}{4 \pi\left|\boldsymbol{C}(\zeta)+\boldsymbol{N}^{u}(\zeta) r_{0}-\widetilde{\boldsymbol{C}}\left(\zeta^{\prime}\right)\right|}$

Here $k=\omega / c$ is the wave number and $r_{0}$ the wire radius.

For the solutions of Eqs. (1) and (2) it is known (Nitsch et al., 2009) that the current $i$ fullfills a second-order ODE of the form

$\left(\frac{\partial^{2}}{\partial \zeta^{2}}+j \omega P_{11}(\zeta) \frac{\partial}{\partial \zeta}+\omega^{2} P_{12}(\zeta)\right) i(\zeta)=0$.

Together with the continuity Eq. (1) the wave-propagation Eq. (7) can be transformed into the TLST equation of the form

$\frac{\partial}{\partial \zeta}\left[\begin{array}{c}q \\ i\end{array}\right]+j \omega \underbrace{\left[\begin{array}{cc}P_{11} & P_{12} \\ 1 & 0\end{array}\right]}_{:=\overline{\mathbf{P}}}\left[\begin{array}{l}q \\ i\end{array}\right]=\left[\begin{array}{l}0 \\ 0\end{array}\right]$.

This is a coupled first-order ODE for the per-unit length charge $q$ and the current $i$. The general solution can be expressed in terms of the matrizant $\mathcal{M}$ (Gantmacher, 1984) as

$\left[\begin{array}{l}q\left(\zeta^{\prime}\right) \\ i\left(\zeta^{\prime}\right)\end{array}\right]=\mathcal{M}_{\zeta}^{\zeta^{\prime}}\{-j \omega \overline{\mathbf{P}}\}\left[\begin{array}{c}q(\zeta) \\ i(\zeta)\end{array}\right]$

The calculation of the per-unit length parameter matrix $\overline{\mathbf{P}}$ is done numerically by an iteration process described in detail in (Rambousky et al., 2012b). Due to experiences made so far with different cable configurations and comparisons with experimental and numerical (e.g., Method of Moments codes) results, already the first iteration parameter matrix, $\overline{\mathbf{P}}^{(1)}$ is accurate enough (Rambousky et al., 2012a).

The so far used charge-current representation for the TLST equation is advantageous for performing the iteration process but it is difficult to determine the boundary conditions for the charge distribution. Voltage and current are fundamental quantities that can be measured easily. Therefore, the potential-current representation for the parameter matrix, denoted by $\overline{\mathbf{P}}^{*}$ is more appropriate. It has to be considered that potential is not a uniquely defined quantity and generally does not equal voltage. However, it is assumed that at least at the ends of the TL potential almost equals voltage. After the first iteration step is performed in charge-current representation, the TLST parameter matrix in potential-current representation, $\overline{\mathbf{P}}^{*(1)}$, can be calculated from $\overline{\mathbf{P}}^{(1)}$ and inserted into the corresponding TLST equation

$$
\frac{\partial}{\partial \zeta}\left[\begin{array}{c}
\varphi \\
i
\end{array}\right]+j \omega \underbrace{\left[\begin{array}{ll}
P_{11}^{*(1)} & P_{12}^{*(1)} \\
P_{21}^{*(1)} & P_{22}^{*(1)}
\end{array}\right]}_{:=\overline{\mathbf{P}}^{*(1)}}\left[\begin{array}{l}
\varphi \\
i
\end{array}\right]=\left[\begin{array}{l}
0 \\
0
\end{array}\right] .
$$


The general solution of Eq. (10) then is

$\left[\begin{array}{c}\varphi\left(\zeta^{\prime}\right) \\ i\left(\zeta^{\prime}\right)\end{array}\right]=\mathcal{M}_{\zeta}^{\zeta^{\prime}}\left\{-j \omega \overline{\mathbf{P}}^{*(1)}\right\}\left[\begin{array}{c}\varphi(\zeta) \\ i(\zeta)\end{array}\right]$.

In our example the boundary value conditions are given by the generator voltage, $U_{0}$, the generator's internal impedance, $Z_{0}$, and a varying terminating load impedance, $Z_{L}$. The relationship between these values and the potential and current values at the beginning and the end of the TL is expressed by

$\varphi(0)=U_{0}-Z_{0} \cdot i(0)$

$\varphi(L)=Z_{L} \cdot i(L)$

After transferring that boundary value problem into the Cauchy problem Eq. (11) the current on the TL can be easily determined.

\section{Transmission-line super theory analysis}

\subsection{Super theory transmission-line parameters}

In TLST the transmission-line parameters $\overline{\mathbf{P}}^{*}$ become complex valued and position and frequency dependent for the first and higher iterations. For a single wire TL the parameter matrix is a 2 by 2 matrix and is now fully occupied. In classical TLT the parameter matrix is off-diagonal and real valued and the $(1,2)$ element is the per-unit length inductance and the $(2,1)$ element the per-unit length capacitance. However, for the first and higher iterations in TLST the parameter matrix elements have no direct physical meaning (Nitsch and Tkachenko, 2009). The reason is that they depend on the chosen gauge (e.g., Lorenz gauge, Coulomb gauge) and the fact that potential is not a unique quantity. Only for a pure TEMmode the voltage between two points on the transmissionline is just the difference between the potentials.

Figure 2 shows the real part of the $(1,2)$ element of the parameter matrix $\overline{\mathbf{P}}^{*(1)}$ for several frequencies. The position and frequency dependency resulting from the non-uniformity of the TL can clearly be seen. The imaginary part of the $(2,1)$ element is depicted in the same way in Fig. 3. As the imaginary parts of $P_{12}^{*(1)}$ and $P_{21}^{*(1)}$ are a kind of measure of the radiated losses of the TL, Fig. 3 shows the increasing radiation of the TL with increasing frequency.

\subsection{Definition of a TLST characterstic impedance}

In classical TLT the characteristic impedance, $Z_{C}$, of the TL is calculated using the per-unit length inductance, $L^{\prime}$, and the per-unit length capacitance, $C^{\prime}$ as

$Z_{C}=\sqrt{\frac{L^{\prime}}{C^{\prime}}}$.

Although having in mind that for TLST the elements of the off-diagonal elements of the parameter matrix have a priori not the physical meaning of a per-unit length inductance

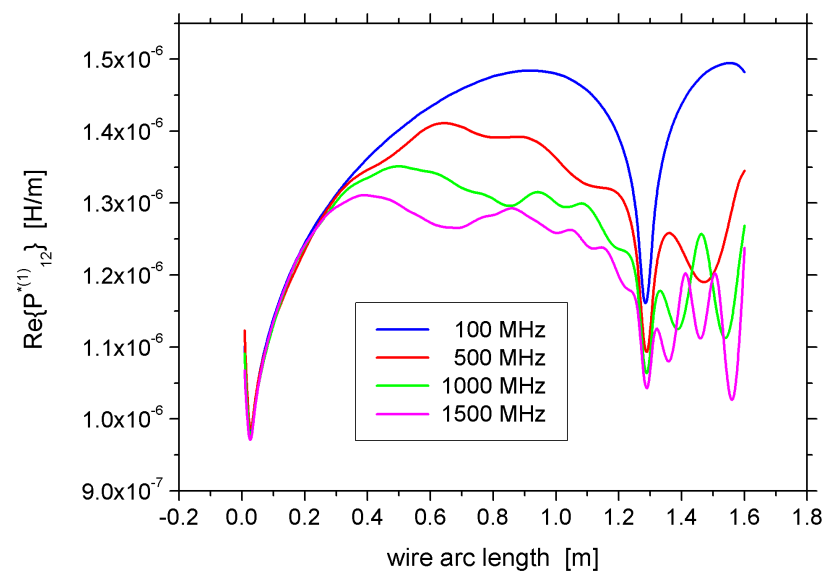

Fig. 2. Real part of $P_{12}^{*(1)}$ for the frequencies of $100 \mathrm{MHz}, 500 \mathrm{MHz}$, $1000 \mathrm{MHz}$ and $1500 \mathrm{MHz}$.



Fig. 3. Imaginary part of $P_{21}^{*(1)}$ for the frequencies of $100 \mathrm{MHz}$, $500 \mathrm{MHz}, 1000 \mathrm{MHz}$ and $1500 \mathrm{MHz}$.

and capacitance it is possible to define a TLST characteristic impedance, $Z_{C}^{\mathrm{TLST}}$ likewise to Eq. (14) as

$Z_{C}^{\mathrm{TLST}}:=\operatorname{Re}\left\{\sqrt{P_{12}^{*(1)} \cdot\left(P_{21}^{*(1)}\right)^{-1}}\right\}$.

In our case the imaginary part of $\sqrt{P_{12}^{*(1)} \cdot\left(P_{21}^{*(1)}\right)^{-1}}$ is smaller than $15 \%$ compared to the real part on the whole line and for each regarded frequency. Therefore, with Eq. (15) we define our $Z_{C}^{\text {TLST }}$ as a real value which also corresponds to the classical definition of the characteristic impedance $Z_{C}$. The physical unit of both $Z_{C}$ and $Z_{C}^{\mathrm{TLST}}$ is the Ohm.

For the frequencies of $100 \mathrm{MHz}, 500 \mathrm{MHz}, 1000 \mathrm{MHz}$ and $1500 \mathrm{MHz}$ the position dependent values of $Z_{C}^{\mathrm{TLST}}$ are shown in Fig. 4. The higher the frequency the lower is the maximum value of $Z_{C}^{\text {TLST }}$ and the more oscillations occur mainly 




Fig. 4. Real part of the TLST characteristic impedance, $Z_{C}^{\mathrm{TLST}}$, for the frequencies of $100 \mathrm{MHz}, 500 \mathrm{MHz}, 1000 \mathrm{MHz}$ and $1500 \mathrm{MHz}$.

around the upper bend of the TL. It has to be mentioned that the calculation of the transmission-line parameters and the TLST characteristic impedance is independent of the choice of the generator and the terminating impedance.

\subsection{Finding an optimal terminating impedance}

In classical TLT the characteristic impedance is a real valued constant. A terminating load with that characteristic impedance is called a matched load. In that case there is no reflection of the forward traveling wave in the load at the end of the TL and therefore no standing waves on the TL. Having in mind that for non-uniform transmission lines there will not exist a perfect matched situation the task for finding an optimal terminating impedance is minimizing the variation of the current on the TL.

Regarding the position and frequency dependent TLST characteristic impedance of Fig. 4 three possibilities are striking for a first choice of the terminating impedance: the value of $Z_{C}^{\mathrm{TLST}}$ at the end of the line, the maximum value or the average value of $Z_{C}^{\mathrm{TLST}}$ over the TL.

For the following analysis a generator with a voltage of $1 \mathrm{~V}$ and an internal impedance of $50 \Omega$ was taken. With $U_{0}=$ $1 V, Z_{0}=50 \Omega$ and the terminating impedance $Z_{L}$ the TLST equation can be solved using

$$
\left[\begin{array}{c}
\varphi(\zeta, k) \\
i(\zeta, k)
\end{array}\right]=\mathcal{M}_{0}^{\zeta}\left\{-j \omega \overline{\mathbf{P}}^{*(1)}(\zeta, k)\right\}\left[\begin{array}{c}
\varphi(0, k) \\
i(0, k)
\end{array}\right]
$$

The wave number is defined by $k=2 \pi f / c$ with $f$ being the frequency and $c$ the propagation velocity of electromagnetic waves on the TL.

For a frequency of $500 \mathrm{MHz}$ the end point value of $Z_{C}^{\mathrm{TLST}}$ on the line is $270 \Omega$, the mean value $391 \Omega$ and the maximum value $424 \Omega$. For these values of $Z_{C}^{\text {TLST }}$ and additionally for



Fig. 5. Current on the TL for a frequency of $500 \mathrm{MHz}$ and different terminating impedances, $Z_{L}$.

the short circuited case and $1000 \Omega$ the modulus of the current on the TL is depicted in Fig. 5. It is clearly seen that the probably intuitive choice of the end point value of $Z_{C}^{\mathrm{TLST}}$ does not minimize the variation of the current on the TL. The least variation of the current on the TL is achieved with a terminating impedance with the maximum value of $Z_{C}^{\mathrm{TLST}}$. This clearly indicates that matching is not a local effect at the position of the concentrated load but a cooperative process including the whole wire.

In Fig. 6 is shown that the above argumentation also holds for a much higher frequency of $1500 \mathrm{MHz}$. However, because of the frequency dependency of the TLST parameters, the end point value of $Z_{C}^{\mathrm{TLST}}$ on the line is now $256 \Omega$, the mean value $367 \Omega$ and the maximum value $396 \Omega$. For broadband applications there will be no single optimal terminating impedance for all frequencies. An averaged value of the different maximum values of $Z_{C}^{\mathrm{TLST}}$ over the TL for the desired frequency range should be taken in such a case.

\subsection{Current in the terminating load}

The frequency behavior of the current in the terminating load is investigated for the frequency range between $100 \mathrm{MHz}$ and $1500 \mathrm{MHz}$. As stated before the maximum, mean and end point value of $Z_{C}^{\text {TLST }}$ varies over frequency. This is shown in Fig. 7 for the maximum value over the TL, denoted as $Z_{C}^{\max }$. The averaged value of $Z_{C}^{\max }$ over the considered frequency range is about $420 \Omega$ which corresponds to a frequency of about $550 \mathrm{MHz}$. So the different terminating loads for the previous used frequency of $500 \mathrm{MHz}$ (see Fig. 5) can be taken for a first proof of concept.

Figure 8 shows the current in the terminating load of the non-uniform TL for the mentioned choices of $Z_{L}$. The different pronounced variations can clearly be seen. Of course, the 


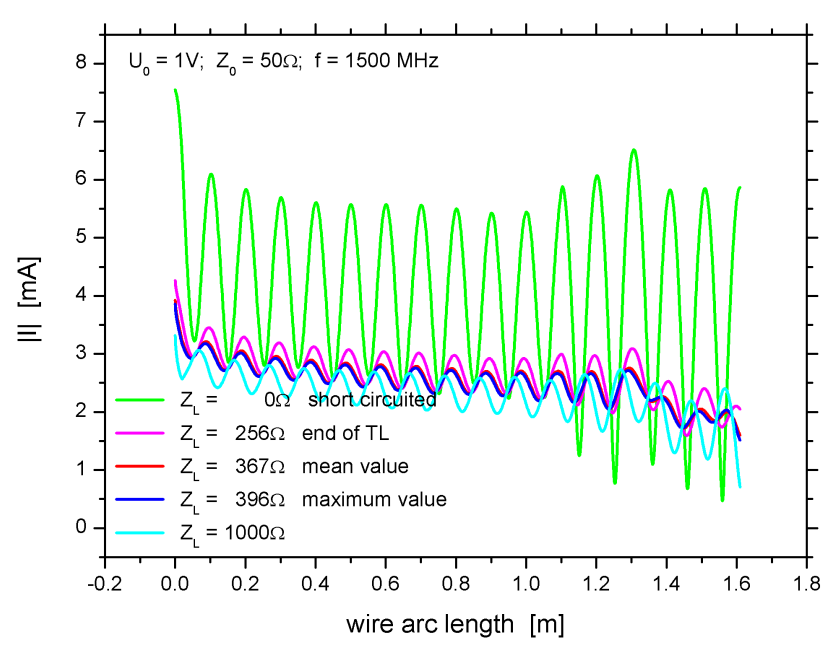

Fig. 6. Current on the TL for a frequency of $1500 \mathrm{MHz}$ and different terminating impedances, $Z_{L}$



Fig. 7. Frequency dependence of the maximal value of the TLST characteristic impedance, $Z_{C}^{\mathrm{TLST}}$

current itself declines with higher values of $Z_{L}$. Although the maximum value of $Z_{C}^{\mathrm{TLST}}$ is not constant over the frequency range we can recognize the lowest variation in the terminating load current. It has to be mentioned that using the mean value of $Z_{C}^{\mathrm{TLST}}$ also gives a relatively small variation of the current.

\subsection{Radiated power of the TL}

The averaged power radiated from a non-uniform transmission-line generally can be obtained by the difference of the power, which is fed into the TL at the beginning and the power which arrives at the terminating load. In (Nitsch and Tkachenko, 2010; Rambousky et al.,



Fig. 8. Frequency dependence of the current in the terminating load for different proposed values of the terminating load, $Z_{L}$

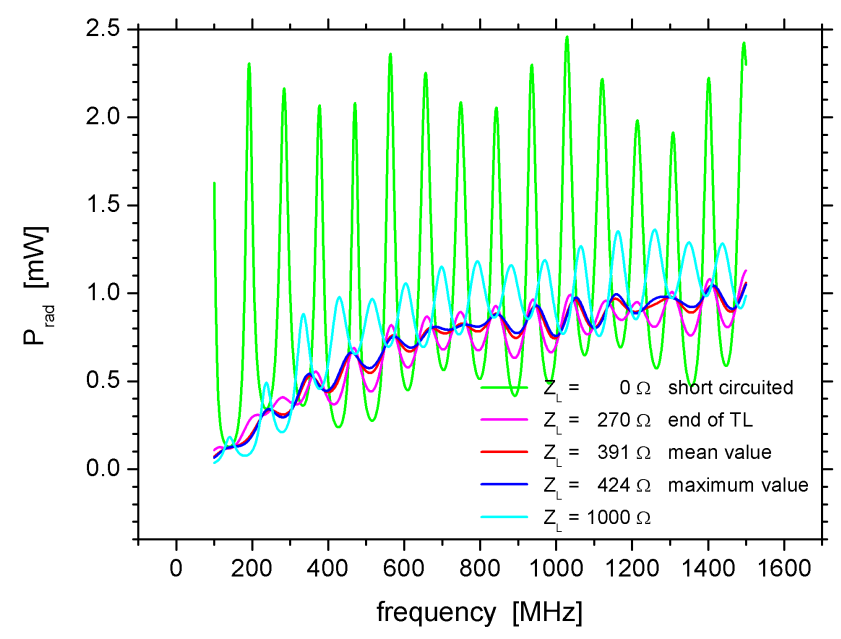

Fig. 9. Frequency dependence of the radiated power for different proposed values of the terminating load, $Z_{L}$

$2012 b$ ) it is shown that for a single wire TL the averaged radiated power can be calculated from the complex valued transmission-line parameters according to

$$
\begin{aligned}
P_{\text {rad }} & =-\frac{\omega}{2} \int_{0}^{L}\left[\operatorname{Im}\left(P_{12}^{*(1)}\right)|i|^{2}+\operatorname{Im}\left(P_{21}^{*(1)}\right)|\varphi|^{2}\right. \\
& \left.+\operatorname{Im}\left(\varphi\left(P_{11}^{*(1)}-P_{22}^{*(1) \dagger}\right) i^{\dagger}\right)\right] d \zeta .
\end{aligned}
$$

In Eq. (17) $P_{22}^{*(1) \dagger}$ and $i^{\dagger}$ denote the complex conjugate of the matrix element $P_{22}^{*(1)}$ and the current $i$, respectively.

The radiated power of the non-uniform TL for the different terminating loads of Sect. 4.4 is shown in Fig. 9. Again one 
can notice that a terminating load corresponding to the maximum value of $Z_{C}^{\mathrm{TLST}}$ minimizes the variation of $P_{\text {rad }}$ in the considered frequency range. The mean value of $Z_{C}^{\mathrm{TLST}}$ also leads to a very good reduction of variation in the radiated power but already the end point value shows a significant enhancement.

\section{Conclusions}

On a non-uniform TL the non-uniformity itself produces reflections all over the TL. In this respect it is not possible to have absolutely no reflections on the line as it is known from classical TLT in the matched state. Therefore, a matched nonuniform TL can be regarded as a TL with minimum variation of the current caused by the right choice of the terminating impedance. In this sense matching is not a local effect at the TL's end but a cooperative process involving the whole nonuniform TL. It could be shown that with the TLST parameters a kind of characteristic impedance can be defined which is position and frequency dependent. Taking the real part of the maximum value of that TLST characteristic impedance as terminating load of the TL, the variation of the current on the line can be minimized.

Although the so defined TLST characteristic impedance is frequency dependent an averaged value for a desired frequency range can be taken as a fixed terminating load. It could be shown that such a choice also minimizes the variation of the current in the terminating load and the radiated power in that frequency range.

The concept of defining a TLST characteristic impedance can be extended to multi-wire transmission-lines. In this case $\mathbf{P}_{12}^{*(1)}$ and $\mathbf{P}_{21}^{*(1)}$ become matrices. The definition of $Z_{C}^{\text {TLST }}$ like in (15) can also be achieved in matrix notation using the inverse of $\mathbf{P}_{21}^{*(1)}$ and performing the root operation on the matrix product $\mathbf{P}_{12}^{*(1)} \cdot \mathbf{P}_{21}^{*(1)^{-1}}$ by means of matrix diagonalization. Future work has to show whether the proposed concept is applicable to non-uniform multi-wire transmission-lines.
Acknowledgements. The authors would like to thank S. Tkachenko for helpful discussions.

\section{References}

Gantmacher, F.: The theory of matrices, Chelsea Publishing Company, New York, 1984.

Haase, H.: Full-Wave Interactions of Nonuniform Transmission Lines, in: Res Electricae Magdeburgenses (MAFO Vol. 9), edited by: Nitsch, J. and Styczynski, Z., Magdeburg, 2005.

Haase, H. and Nitsch, J.: Full-wave transmission-line theory (FWTLT) for the analysis of three dimensional wire-like structures, in: Proc. 14th International Zurich Symposium and Technical Exhibition on Electromagnetic Compatibility, 235-240, Zurich, Switzerland, 2001.

Haase, H., Nitsch, J., and Steinmetz, T.: Transmission-Line Super Theory: A New Approach to an Effective Calculation of Electromagnetic Interactions, Radio Sci. Bull., 307, 33-60, 2003.

Nitsch, J. and Tkachenko, S.: Physical Interpretation of the Parameters in the Full-Wave Transmission Line Theory, in: Proc. VXV. International Symposium on Theoretical Engineering, 3034, Luebeck, Germany, 2009.

Nitsch, J. and Tkachenko, S.: High-Frequency Multiconductor Transmission-Line Theory, Found. Phys., 40, 1231-1252, 2010.

Nitsch, J., Gronwald, F., and Wollenberg, G.: Radiating Nonuniform Transmission-Line Systems and the Partial Element Equivalent Circuit Method, Wiley, Chichester, West Sussex, UK, 2009.

Rambousky, R., Nitsch, J., and Garbe, H.: Analyzing Simplified Open TEM-Waveguides using Transmission-Line Super Theory, in: Proc. EMC Europe 2012, Rome, Italy, 2012a.

Rambousky, R., Nitsch, J., and Garbe, H.: Application of Transmission-Line Super Theory to Multi-Wire TEMWaveguide Structures, IEEE Trans. Electromagn. Compat., submitted for publication, $2012 \mathrm{~b}$.

Tesche, F.: The effect of the thin-wire approximation and the source gap model on the high frequency integral equation solution of radiating antennas, IEEE Trans. Antennas Propag., A-20, 210211, 1972. 